\title{
VORTRÄGE Begutachtung in der Pneumologie
}

Presentations

tung der berufsspezifischen Allergene in krankheitsauslösender Form auf dem Arbeitsmarkt ab. Konkret bedeutet eine MdE-Einschätzung von 10 von Hundert, dass dem Betroffenen 10\% der Arbeitsmöglichkeiten durch seine anerkannte Berufskrankheit verschlossen sind.

Probleme treten häufig bei der MdE-Einschätzung auf. Der irritative Schaden am Hautorgan, der zu einer Minderbelastbarkeit auch nach Aufgabe der schädigenden Tätigkeit führt, bedarf einer fallbezogenen Konkretisierung. Nicht immer kommt es zur Restauration eines irritativen Haut- schadens; trotz fehlender ärztlicher Behandlungen treten Hautschäden nach mehr oder minder starken alltäglichen Belastungen der Haut in Erscheinung und zwingen den Betroffenen zur Meidung der auslösenden Tätigkeiten. Die Verbreitung der Allergene in krankheitsauslösender Form wird häufig kontrovers eingeschätzt. Insbesondere bei einem kombinierten Vorliegen von Haut- und Schleimhauterkrankungen kommt es bei der Gesamtbewertung zu divergierenden Einschätzungen (Beispiel Mehlstauballergien bei Bäckern, Latexallergien im Gesundheitswesen).

\section{Literatur}

1. Blome O. Neufassung der Empfehlungen für die Einschätzung der Minderung der Erwerbsfähigkeit bei Berufskrankheiten der Haut nach BK 5101 der Anlage 1 zur Berufskrankheiten-Verordnung. Dermatosen 1998; 46: 29-33

2. Schönberger A, Mehrtens G, Valentin H. Arbeitsunfall und Berufskrankheit. Rechtliche und medizinische Grundlagen für Gutachter, Sozialverwaltung, Berater und Gerichte. Berlin: Erich Schmidt Verlag, 1998
3. Mehrtens G, Perlebach E. Die Berufskrankheiten- verordnung (BeKV), Kommentar. Loseblattwerk, Erich Schmidt Verlag, Berlin

\author{
Priv.-Doz. Dr. Wolfgang Wehrmann \\ Warendorfer Straße183 \\ 48145 Münster
}

\section{Begutachtung in der Pneumologie}

\section{WERNER KERSTEN}

Im Rahmen der Präsentation werden die Berufserkrankungen 4301/4302 und 4201 und 1315 abgehandelt. Für diese berufsbedingten Atemwegserkrankungen werden die Besonderheiten bei der Anamneseerhebung, den Hauttests und den organbezogenen Provokationstests dargestellt.

Auch serologische Untersuchungstechniken spielen bei der Diagnostik beruflicher Atemwegserkrankungen eine immer größere Rolle. Die Palette reicht von Bestimmungen spezifischer IgE- und IgG-Antikörper sowie präzipitierender Antikörper bis zur Anwendung von CAST - ECP - CRIE oder Immunoblot-Untersuchungen. Für einige Krankheitsbilder ist der primäre serologische Nachweis von spezifischen Antikörpern vor der Durchführung von Hauttests sinnvoll. Die RAST-Inhibition dient zur Klärung der Frage, ob eine Kreuzreagibilität vorliegt oder ob es sich um eine eigenständige Sensibilisierung z. B. auf Mehle handelt.
Eigene Ergebnisse von Untersuchungen von Versicherten im Bäckerei-, Frisör-, Schreinereihandwerk, bei Malern und Lackierern und bei Schleifern werden vorgestellt; die Untersuchungsmöglichkeiten und Spezialitäten bei anderen Berufsgruppen wie Landwirten, Schweißern, Floristen usw. werden abgehandelt.

Nach gesicherter Diagnostik können mehrere therapeutische Maßnahmen ergriffen werden. Eine häufige Konsequenz ist die Aufgabe des Berufes. Die verschiedenen Möglichkeiten, die die BeKV den Versicherten bietet, werden dargestellt.

\author{
Dr. med. Werner Kersten \\ Institut für Umwelt- und Arbeitsmedizin \\ Uerdinger Straße 3 \\ 47441 Moers
}

